\title{
Security and composability of randomness expansion from Bell inequalities
}

\author{
Serge Fehr, ${ }^{1}$ Ran Gelles, ${ }^{2}$ and Christian Schaffner ${ }^{1,3}$ \\ ${ }^{1}$ Centrum Wiskunde \& Informatica, Amsterdam, Netherlands \\ ${ }^{2}$ Department of Computer Science, University of California, Los Angeles, California 90095, USA \\ ${ }^{3}$ Institute for Logic, Language and Computation (ILLC), University of Amsterdam, Amsterdam, Netherlands
}

(Received 20 September 2012; published 30 January 2013)

\begin{abstract}
The nonlocal behavior of quantum mechanics can be used to generate guaranteed fresh randomness from an untrusted device that consists of two nonsignalling components; since the generation process requires some initial fresh randomness to act as a catalyst, one also speaks of randomness expansion. R. Colbeck and A. Kent [J. Phys. A 44, 095305 (2011)] proposed the first method for generating randomness from untrusted devices, but without providing a rigorous analysis. This was addressed subsequently by S. Pironio et al. [Nature (London) 464, 1021 (2010)], who aimed at deriving a lower bound on the min-entropy of the data extracted from an untrusted device based only on the observed nonlocal behavior of the device. Although that article succeeded in developing important tools for reaching the stated goal, the proof itself contained a bug, and the given formal claim on the guaranteed amount of min-entropy needs to be revisited. In this paper we build on the tools provided by Pironio et al. and obtain a meaningful lower bound on the min-entropy of the data produced by an untrusted device based on the observed nonlocal behavior of the device. Our main result confirms the essence of the (improperly formulated) claims of Pironio et al. and puts them on solid ground. We also address the question of composability and show that different untrusted devices can be composed in an alternating manner under the assumption that they are not entangled. This enables superpolynomial randomness expansion based on two untrusted yet unentangled devices.
\end{abstract}

DOI: 10.1103/PhysRevA.87.012335

PACS number(s): 03.67.Dd, 03.65.Ud

\section{INTRODUCTION}

\section{A. Background}

One of the counterintuitive features of quantum mechanics is its nonlocality: measuring possibly far apart quantum systems in randomly selected bases (chosen out of some given class) may lead to correlations that are impossible to obtain classically. Anticipated by Einstein, Rosen, and Podolsky [1], it was Bell [2] who put this property on firm ground by proposing an inequality that is satisfied by any classical correlation but is violated when the correlation is obtained from measuring entangled quantum states. Such inequalities are called Bell inequalities.

An important example of such a Bell inequality was proposed by Clauser, Horne, Shimony, and Holt (CHSH) [3] and states that if $X$ and $Y$ are independent uniformly distributed bits and if bit $A$ is obtained by "processing" $X$ without knowing $Y$ and bit $B$ is obtained by "processing" $Y$ without knowing $X$, then the probability that $A \oplus B=X \wedge Y$ is at most $75 \%$. This bound on the probability holds if the processing is done classically with shared randomness but can be violated when the processing involves measuring an entangled quantum state; in this latter case, a probability of roughly $85 \%$ can be achieved.

Violating a Bell inequality necessarily means that there must be some amount of fresh randomness in the outputs $A$ and $B$ (given the inputs $X$ and $Y$ ). More formally, consider an

Published by the American Physical Society under the terms of the Creative Commons Attribution 3.0 License. Further distribution of this work must maintain attribution to the author(s) and the published article's title, journal citation, and DOI. untrusted device $\mathfrak{D}$, prepared by an adversarial manufacturer Eve. The device consists of two components, set up by Eve, which on respective inputs $X$ and $Y$ produce respective outputs $A$ and $B$ without communicating. No matter how the two components work, as long as a given Bell inequality is violated during $n$ sequential interactions with $\mathfrak{D}$ (which can be observed by doing statistics), there must be a certain amount of uncertainty in the $n$ output pairs $\left(A_{1}, B_{1}\right), \ldots,\left(A_{n}, B_{n}\right)$, even given the $n$ input pairs $\left(X_{1}, Y_{1}\right), \ldots,\left(X_{n}, Y_{n}\right)$, and thus it should be possible to apply a randomness extractor to obtain nearly random bits.

This kind of randomness expansion from untrusted devices was first suggested by Colbeck [4] and Colbeck and Kent [5], who presented a scheme that uses Greenberger-HorneZeilinger (GHZ) states and reaches a linear expansion, but without providing a rigorous security analysis. The main point missing in these works is a method to rigorously bound the min-entropy of a device's output. The work of Pironio et al. [6] addresses this issue, and they propose a technique to numerically compute a lower bound on the min-entropy of the output pair $A B$ (conditioned on $X$ and $Y$ ) as a function of the Bell value of the device $\mathfrak{D}$ (which quantifies the violation of Bell inequality). For the special case of CHSH, they also show an analytical bound.

The authors of [6] also consider the case of $n$ sequential interactions with $\mathfrak{D}$, and they show how to estimate the average Bell value of $\mathfrak{D}$ over $n$ rounds by doing statistics over the observed data. This is nontrivial because the Bell value of $\mathfrak{D}$ may change over the different rounds, and for each round, it may depend on the behavior of the previous rounds. In other words, the Bell value of $\mathfrak{D}$ during round $i+1$ depends on the history $\left(A_{1}, B_{1}, X_{1}, Y_{1}\right), \ldots,\left(A_{i}, B_{i}, X_{i}, Y_{i}\right)$. Pironio et al. then claim to have a bound on the min-entropy 
of $\left(A_{1}, B_{1}\right), \ldots,\left(A_{n}, B_{n}\right)$ conditioned on $\left(X_{1}, Y_{1}\right), \ldots,\left(X_{n}, Y_{n}\right)$, where the bound is actually a function of the observed data, i.e., a function of $\left(A_{1}, B_{1}, X_{1}, Y_{1}\right), \ldots,\left(A_{n}, B_{n}, X_{n}, Y_{n}\right)$.

However, the claimed bound in [6] does not hold in general; there is a flaw in its derivation, which is without an obvious fix. ${ }^{1}$ Thus, even though the necessary tools are provided in [6], they are not put together in the right way to be able to control the min-entropy of $\left(A_{1}, B_{1}\right), \ldots,\left(A_{n}, B_{n}\right)$ produced by an untrusted device $\mathfrak{D}$.

\section{B. Our result}

In this paper, we make up for this shortfall in [6]. Specifically, we put together the tools provided in [6] in order to obtain a correct bound on the min-entropy of $\left(A_{1}, B_{1}\right), \ldots,\left(A_{n}, B_{n}\right)$, conditioned on $\left(X_{1}, Y_{1}\right), \ldots,\left(X_{n}, Y_{n}\right)$, by means of the observed data. The trick is to consider and bound the min-entropy conditioned on the event that the estimator for the average Bell value lies in some interval. This gives us some control over the average Bell value of the device but, as we show, still leaves enough uncertainty in the data to get a good bound on its min-entropy.

We also address the question of the composability of untrusted devices. We show that under the assumption that different devices are not entangled, the output of one device, after privacy amplification, can be used as the input for a second device, and the resulting output of the second device, after privacy amplification, can again be fed into the first device and so on. Using an extractor with a short seed to do the privacy amplification, this allows for a superpolynomial randomnessexpansion scheme using two untrusted (but guaranteed to be unentangled) devices.

\section{Concurrent and related work}

In concurrent and independent work, Vazirani and Vidick [7] as well as Pironio and Massar [8] came up with results that overlap with ours. We briefly discuss here the similarities and the differences between our results and those of Vazirani and Vidick and of Pironio and Massar. We encourage the reader to also look at the comparisons given in [7,8].

Vazirani and Vidick obtain a randomness-expansion scheme with superpolynomial expansion and security against quantum side information. We do not achieve security against quantum side information, and our superpolynomial randomness-expansion scheme requires two unentangled devices in an iterative way, whereas their scheme works with just one single device. On the other hand, their result is tailored to $\mathrm{CHSH}$, while our result is generic and holds for any Bell

\footnotetext{
${ }^{1}$ As a matter of fact, the formal statement is already suspicious since the min-entropy is a fixed number, determined by the underlying probability distribution, whereas the claimed bound is a random variable. This is like saying that we can lower bound the min-entropy of throwing a fair die by the result of the throw. The former equals $\log _{2}(6) \approx 2.6$, whereas the latter is a random number in $\{1, \ldots, 6\}$. We also point out that trying to bound the min-entropy conditioned on the observed outcome makes no sense either because this conditional min-entropy obviously vanishes.
}

inequality. Pironio and Massar's results, on the other hand, are very similar to ours and only differ in some minor details. ${ }^{2}$

In a very recent paper, Barrett et al. point out the possibility of Trojan-horse attacks on device-independent randomnessexpansion protocols (see the Supplemental Material of [9]). It seems impossible to prevent Eve from programming devices (that are used multiple times) to release in later rounds information about previous outputs. We note that although such an attack seems unavoidable, in a single activation of our randomness-expansion scheme (see Sec. IV for details), we can reuse the same devices over and over again and still prevent such a Trojan-horse attack by only releasing the output of the very last round (and aborting if things go wrong before the last round is reached).

\section{PRELIMINARIES}

We assume the reader is familiar with quantum information processing, and we merely fix our notation and some basic concepts in this section.

\section{A. Quantum states}

The state of a quantum system $\mathcal{A}$ is given by a density matrix $\rho_{\mathcal{A}}$, i.e., a positive-semidefinite trace- 1 matrix acting on some Hilbert space $\mathcal{H}_{\mathcal{A}}$. We denote the set of all such matrices, acting on $\mathcal{H}_{\mathcal{A}}$, by $D\left(\mathcal{H}_{\mathcal{A}}\right)$. The state space of the joint quantum systems $\mathcal{A B}$, which consist of two (or more) subsystems $\mathcal{A}$ and $\mathcal{B}$, is given by the tensor product $\mathcal{H}_{\mathcal{A B}}=\mathcal{H}_{\mathcal{A}} \otimes \mathcal{H}_{\mathcal{B}}$. If the state of the joint system is given by $\rho_{\mathcal{A}}$, then the state of subsystem $\mathcal{A}$ when considered as a "stand-alone" system is given by the reduced density matrix $\rho_{\mathcal{A}}=\operatorname{tr}_{\mathcal{B}}\left(\rho_{\mathcal{A B}}\right) \in D\left(\mathcal{H}_{\mathcal{A}}\right)$, obtained by tracing out system $\mathcal{B}$.

A random variable $X$ over a finite set $\mathbb{X}$ with probability distribution $P_{X}$ can be represented by means of the density matrix as $\rho_{X}=\sum_{X} P_{X}(x)|x\rangle\langle x| \in D\left(\mathcal{H}_{X}\right)$, where $\{|x\rangle\}_{x \in \mathbb{X}}$ forms a basis of $\mathcal{H}_{X}=\mathbb{C}^{|\mathbb{X}|}$. Thus, we may view $X$ as a quantum system, and we say that its state, $\rho_{X}$, is classical. If the state of a quantum system $\mathcal{E}$ depends on the random variable $X$, in that the state of $\mathcal{E}$ is given by $\rho_{\mathcal{E}}^{x} \in D\left(\mathcal{H}_{\mathcal{E}}\right)$ if $X=x$, then we can view the pair $X \mathcal{E}$ as a bipartite quantum system in state $\rho_{X \mathcal{E}}=\sum_{x} P_{X}(x)|x\rangle\langle x| \otimes \rho_{\mathcal{E}}^{x} \in D\left(\mathcal{H}_{X} \otimes \mathcal{H}_{\mathcal{E}}\right)$. This naturally extends to multiple random variables and quantum systems.

The distance between two states $\rho_{\mathcal{E}}, \tilde{\rho}_{\mathcal{E}} \in D\left(\mathcal{H}_{\mathcal{E}}\right)$ is measured by their trace distance $\frac{1}{2}\left\|\rho_{\mathcal{E}}-\tilde{\rho}_{\mathcal{E}}\right\|_{1}$, where $\|\cdot\|_{1}$ is the $L_{1}$ norm. ${ }^{3}$ In case of classical states $\rho_{X}$ and $\tilde{\rho}_{X}$, corresponding to distributions $P_{X}$ and $\tilde{P}_{X}$, the trace distance coincides with the statistical distance $\frac{1}{2} \sum_{x}\left|P_{X}(x)-\tilde{P}_{X}(x)\right|$.

\section{B. Closeness to uniform, min-entropy, and extractors}

In the following definitions, we consider a bipartite system $X \mathcal{E}$ with classical $X$, given by $\rho_{X \mathcal{E}} . X$ is said to be random

\footnotetext{
${ }^{2}$ As a historical note, previous versions of their paper and our paper claimed security against quantum side information, but both proofs were incorrect.

${ }^{3}$ Defined by $\|A\|_{1}:=\operatorname{tr}\left(\sqrt{A^{\dagger} A}\right)$, where $A^{\dagger}$ denotes the Hermitian transpose.
} 
and independent from $\mathcal{E}$ if $\rho_{X \mathcal{E}}=\rho_{U} \otimes \rho_{\mathcal{E}}$, where $\rho_{U}$ is the fully mixed state on $\mathcal{H}_{X}$ (i.e., $U$ is classical and, as a random variable, uniformly distributed).

Definition 1. The distance to uniform of $X$ given $\mathcal{E}$ is $d(X \mid \mathcal{E}):=\frac{1}{2}\left\|\rho_{X \mathcal{E}}-\rho_{U} \otimes \rho_{\mathcal{E}}\right\|_{1}$.

If $\Omega$ is some event, determined by the random variable $X$, then $d(X \mid \mathcal{E}, \Omega)$ is naturally defined by means of replacing the distribution $P_{X}$ by $P_{X \mid \Omega}$. The same applies to the next two definitions.

Definition 2. The guessing probability of $X$ given $\mathcal{E}$ is

$$
\operatorname{Guess}(X \mid \mathcal{E}):=\sup _{\left\{M_{x}\right\}_{x}} \sum_{x} P_{X}(x) \operatorname{tr}\left(M_{x} \rho_{\mathcal{E}}^{x}\right),
$$

where the supremum is over all positive operator-valued measures $\left\{M_{x}\right\}_{x}$ on $\mathcal{H}_{\mathcal{E}}$.

Definition 3. The min-entropy of $X$ given $\mathcal{E}$ is

$$
H_{\min }(X \mid \mathcal{E}):=-\log _{2} \operatorname{Guess}(X \mid \mathcal{E}) .
$$

This definition was shown in [10] to coincide with the definition originally introduced by Renner [11], which also coincides with the classical definition of conditional minentropy in the case where $\mathcal{E}$ is classical.

Definition 4. A function Ext : $\{0,1\}^{n} \times\{0,1\}^{d} \rightarrow\{0,1\}^{\xi}$ is a $\left(k, \varepsilon_{\text {ext }}\right)$-strong extractor if, for any bipartite quantum system $X \mathcal{E}$ with classical $X$ and with $H_{\min }(X \mid \mathcal{E}) \geqslant k$ and for a uniform and independent seed $Y$, we have $d(\operatorname{Ext}(X, Y) \mid Y \mathcal{E}) \leqslant \varepsilon_{\text {ext }}$.

Note that we find "extractor against quantum adversaries" too cumbersome a term; thus we just call Ext a (strong) extractor, even though it is a stronger notion than the standard notion of a (strong) extractor.

\section{Bell inequality and CHSH}

For given finite sets $\mathbb{A}, \mathbb{B}, \mathbb{X}, \mathbb{Y}$, consider a conditional probability distribution $P_{A B \mid X Y}$, specified as follows. There exists $\rho_{\mathcal{A B}} \in D\left(\mathcal{H}_{\mathcal{A}} \otimes \mathcal{H}_{\mathcal{B}}\right)$ for an arbitrary (finite) dimensional two-partite quantum system $\mathcal{A B}$ and families of measurements $\left\{M_{x}^{a}\right\}$ and $\left\{N_{y}^{b}\right\}$, indexed by $x \in \mathbb{X}$ and $y \in \mathbb{Y}$, acting on $\mathcal{A}$ and $\mathcal{B}$, and with measurement outcomes $a \in \mathbb{A}$ and $b \in \mathbb{B}$, respectively, such that

$$
P_{A B \mid X Y}(a, b \mid x, y)=\operatorname{tr}\left[\left(M_{x}^{a} \otimes N_{y}^{b}\right) \rho_{\mathcal{A B}}\left(M_{x}^{a} \otimes N_{y}^{b}\right)^{\dagger}\right]
$$

for all $(a, b, x, y) \in \mathbb{A} \times \mathbb{B} \times \mathbb{X} \times \mathbb{Y}$.

Definition 5. For any set $\mathcal{C}=\left\{c_{a b x y}\right\}$ of Bell coefficients, the Bell value of $P_{A B \mid X Y}$ (with respect to $\mathcal{C}$ ) is defined as

$$
I\left(P_{A B \mid X Y}\right)=\sum_{a b x y} c_{a b x y} P_{A B \mid X Y}(a, b \mid x, y) .
$$

$P_{A B \mid X Y}$ is called classical (or local) if there exist (conditional) probability distributions $P_{R}, P_{A \mid X R}$, and $P_{B \mid Y R}$ such that

$P_{A B \mid X Y}(a, b \mid x, y)=\sum_{r} P_{R}(r) P_{A \mid X R}(a \mid x, r) P_{B \mid Y R}(b \mid y, r)$

for all $a, b, x, y$; this is equivalent to requiring that $P_{A B \mid X Y}$ can be specified by means of a separable state $\rho_{\mathcal{A B}}$. We let $I_{0}$ denote the maximal Bell value achievable (for a given set of Bell coefficients) with a classical $P_{A B \mid X Y}$. We speak of a violation of Bell inequality if there exists a quantum system resulting in conditional probability distribution with a Bell value greater than $I_{0}$. For instance, for so-called $\mathrm{CHSH}$ Bell coefficients [3], given by $c_{a b x y}=(-1)^{x y}(-1)^{a \oplus b}$ for $a, b, x, y \in\{0,1\}$, it is known that $I_{0}=2$, but $I=2 \sqrt{2}$ is possible for a quantum system.

\section{FRESH RANDOMNESS FROM UNTRUSTED DEVICES}

In this section, we recall (some of) the findings of [6] and also discuss and fix some subtle issue not discussed there. Throughout this and the following sections, we consider fixed finite sets $\mathbb{A}, \mathbb{B}, \mathbb{X}, \mathbb{Y}$ and a fixed set $\mathcal{C}=\left\{c_{a b x y}\right\}$ of Bell coefficients. The reader may think of $\mathrm{CHSH}$, but our results hold generally.

\section{A. A single interaction}

We consider an untrusted device $\mathfrak{D}$, prepared by an adversary Eve. As discussed in the Introduction, $\mathfrak{D}$ consists of two components, which, on respective inputs $x \in \mathbb{X}$ and $y \in \mathbb{Y}$, produce respective outputs $a \in \mathbb{A}$ and $b \in \mathbb{B}$ without communicating. ${ }^{4}$ Formally, $\mathfrak{D}$ 's behavior is given by an unknown conditional probability distribution $P_{A B \mid X Y}$, which is specified by an unknown quantum state $\rho_{\mathcal{A B}} \in D\left(\mathcal{H}_{\mathcal{A}} \otimes \mathcal{H}_{\mathcal{B}}\right)$ of unknown dimension and unknown families of measurements $\left\{M_{x}^{a}\right\}$ and $\left\{N_{y}^{b}\right\}$, acting on the respective systems $\mathcal{A}$ and $\mathcal{B}$. We are interested in the guaranteed amount of uncertainty in $A$ and $B$ (conditioned on $X$ and $Y$ ) under the promise that $P_{A B \mid X Y}$ has some given Bell value greater than $I_{0}$. This motivates the following definition.

Definition 6. For a given set of Bell coefficients, we define $h_{0}$ to be the function

$$
h_{0}(I)=\inf _{\substack{\mathcal{H}_{\mathcal{A}}, \mathcal{H}_{\mathcal{B}}, \rho_{\mathcal{A B}} \\\left\{M_{x}^{a}\right\},\left\{N_{y}^{b}\right\}}} \min _{\substack{x \in \mathbb{X} \\ y \in \mathbb{Y}}} H_{\min }(A B \mid X=x, Y=y),
$$

where the outer infimum is over all finite-dimensional Hilbert spaces $\mathcal{H}_{\mathcal{A}}$ and $\mathcal{H}_{\mathcal{B}}$, all states $\rho_{\mathcal{A B}} \in D\left(\mathcal{H}_{\mathcal{A}} \otimes \mathcal{H}_{\mathcal{B}}\right)$, and all families of measurements $\left\{M_{x}^{a}\right\}$ and $\left\{N_{y}^{b}\right\}$ such that $P_{A B \mid X Y}(a, b \mid x, y)=\operatorname{tr}\left(M_{x}^{a} \otimes M_{y}^{b} \rho_{\mathcal{A B}} M_{x}^{a \dagger} \otimes M_{y}^{b^{\dagger}}\right)$ has a Bell value of at least $I$. Also, we define $h$ to be the convex closure of $h_{0}$, i.e., the maximal convex function that does not exceed $h_{0}{ }^{5}$

Pironio et al. [6] show that by means of a hierarchy of semidefinite programs (SDPs) [12,13], $h_{0}(I)$ can be numerically computed up to arbitrary precision (by means of a possibly expensive computation). They also show an analytical lower bound of $1-\log _{2}\left(1+\sqrt{2-I^{2} / 4}\right)$ for $h_{0}(I)$ in the case of $\mathrm{CHSH}$, which reaches 1 for $I=I_{\max }=2 \sqrt{2}$ [whereas the numerical calculation gives $h_{0}(2 \sqrt{2}) \approx 1.23$ ] and monotonically decreases to 0 as $I$ goes down to $I_{0}=2$; see Fig. 2 in [6]. Since this lower bound is convex, it is also a

\footnotetext{
${ }^{4}$ The results derived here apply also to devices with three or more components, including the three-component devices used in [5].

${ }^{5}$ Formally, $h(I)=\max f(I)$, where the maximum is over all convex functions $f$, which are upper bounded by $h_{0}$.
} 
lower bound on $h$; we will need this later on. ${ }^{6}$ For now, we can conclude that if an unknown bipartite quantum system (with fixed measurements $\left\{M_{x}^{a}\right\}$ and $\left\{N_{y}^{b}\right\}$ ) is promised to have a CHSH value of $I=2 \sqrt{2}$, then the joint min-entropy in the measurement outcomes $A$ and $B$ is lower bounded by approximately 1.23 bits (one bit if one wants to rely on the analytical bound).

\section{B. Sequential repetitions}

In order to get more uncertainty and in order to be able to estimate the Bell value, we consider a sequential repetition of extracting uncertainty from an untrusted device $\mathfrak{D}$ as above. Informally, rather than interacting with $\mathfrak{D}$ once [i.e., inputting $(x, y) \in \mathbb{X} \times \mathbb{Y}$ and observing $(a, b) \in \mathbb{A} \times \mathbb{B}]$, we interact with $\mathfrak{D} n$ times in sequence by inputting $\left(x_{1}, y_{1}\right) \in \mathbb{X} \times \mathbb{Y}$ and observing $\left(a_{1}, b_{1}\right) \in \mathbb{A} \times \mathbb{B}$, inputting $\left(x_{2}, y_{2}\right) \in \mathbb{X} \times \mathbb{Y}$ and observing $\left(a_{2}, b_{2}\right) \in \mathbb{A} \times \mathbb{B}$, etc. This procedure is formalized as follows.

a. Modeling. We consider an arbitrary but fixed bipartite state $\rho_{\mathcal{A B}} \in D\left(\mathcal{H}_{\mathcal{A}} \otimes \mathcal{H}_{\mathcal{B}}\right)$ of an arbitrary finitedimensional bipartite quantum system $\mathcal{A B}$ and a sequence of $n$ arbitrary but fixed pairs of families of measurements $\left(\left\{M_{x_{1}}^{a_{1}}\right\},\left\{N_{y_{1}}^{b_{1}}\right\}\right), \ldots,\left(\left\{M_{x_{n}}^{a_{n}}\right\},\left\{N_{y_{n}}^{b_{n}}\right\}\right)$.

For each pair, $\left\{M_{x_{j}}^{a_{j}}\right\}$ is a family of measurements, indexed by $x_{j} \in \mathbb{X}$, acting on $\mathcal{A}$, with measurement outcomes $a_{j} \in \mathbb{A}$; similarly, $\left\{N_{y_{j}}^{b_{j}}\right\}$ is a family of measurements acting on $\mathcal{B}$. We allow the two components of the device $\mathfrak{D}$ to communicate between rounds; this is captured by a sequence $U_{2}, \ldots, U_{n}$ of unitary transformations acting on $\mathcal{H}_{\mathcal{A}} \otimes \mathcal{H}_{\mathcal{B}}$, where $U_{j}$ is applied to the (collapsed) state before the $j$ th interaction. For $j \in\{1, \ldots, n\}$, denote with $a^{j}$ the concatenation of the first $j$ rounds $a^{j}=a_{1} \cdots a_{j}$ and do the same for $b, x$, and $y$. Let $A^{j}, B^{j}, X^{j}, Y^{j}$ be the corresponding random variables. To ease notation, we use bold letters as shortcuts for the concatenation of all $n$ rounds, e.g., $\boldsymbol{a}=a^{n}, \boldsymbol{A}=A^{n}$, etc.

Formally, the conditional probability distribution $P_{\boldsymbol{A B} \mid \boldsymbol{X Y}}$ is defined as

$$
P_{\boldsymbol{A} \boldsymbol{B} \mid \boldsymbol{X} \boldsymbol{Y}}(\boldsymbol{a}, \boldsymbol{b} \mid \boldsymbol{x}, \boldsymbol{y})=\prod_{j=1}^{n} P_{A_{j} B_{j} \mid X_{j} Y_{j} T_{j}}\left(a_{j}, b_{j} \mid x_{j}, y_{j}, t_{j}\right),
$$

where $T_{j}=\left(A^{j-1}, B^{j-1}, X^{j-1}, Y^{j-1}\right)$ and $t_{j}=\left(a^{j-1}, b^{j-1}\right.$, $\left.x^{j-1}, y^{j-1}\right)$ denote the transcripts up to round $j-1$, and

$$
\begin{aligned}
& P_{A_{j} B_{j} \mid X_{j} Y_{j} T_{j}}\left(a_{j}, b_{j} \mid x_{j}, y_{j}, t_{j}\right) \\
& \quad=\operatorname{tr}\left[\left(M_{x_{j}}^{a_{j}} \otimes N_{y_{j}}^{b_{j}}\right) \rho_{\mathcal{A B} \mid T_{j}=t_{j}}\left(M_{x_{j}}^{a_{j}} \otimes N_{y_{j}}^{b_{j}}\right)^{\dagger}\right],
\end{aligned}
$$

where $\rho_{\mathcal{A B} \mid T_{j}=t_{j}}$ is inductively defined for $j=1, \ldots, n$ as follows. $\rho_{\mathcal{A B} \mid T_{1}=t_{1}}=\rho_{\mathcal{A B}}$, and for $1 \leqslant j<n$,

$$
\begin{aligned}
& \rho_{\mathcal{A B} \mid T_{j+1}=t_{j+1}} \\
& =U_{j+1} \frac{\left(M_{x_{j}}^{a_{j}} \otimes N_{y_{j}}^{b_{j}}\right) \rho_{\mathcal{A B} \mid T_{j}=t_{j}}\left(M_{x_{j}}^{a_{j}} \otimes N_{y_{j}}^{b_{j}}\right)^{\dagger}}{P_{A_{j} B_{j} \mid X_{j} Y_{j} T_{j}}\left(a_{j}, b_{j} \mid x_{j}, y_{j}, t_{j}\right)} U_{j+1}^{\dagger}
\end{aligned}
$$

\footnotetext{
${ }^{6}$ Actually, the numerical computations for CHSH suggest that $h=$ $h_{0}$; we do not know if this holds generally.
}

is the state obtained by applying $U_{j+1}$ to the state to which $\rho_{\mathcal{A B} \mid T_{j}=t_{j}}$ collapses when $\mathcal{A}$ and $\mathcal{B}$ are measured by $\left\{M_{x_{j}}^{a_{j}}\right\}$ and $\left\{N_{y_{j}}^{b_{j}}\right\}$, respectively, and $a_{j}$ and $b_{j}$ are observed.

What is important to realize is that before every round $j$, the situation is exactly as in Sec. III A, with a fixed state $\rho_{\mathcal{A B} \mid T_{j}=t_{j}}$ and fixed measurements $\left\{M_{x_{j}}^{a_{j}}\right\}$ and $\left\{N_{y_{j}}^{b_{j}}\right\}$ in the device $\mathfrak{D}$, and thus $P_{A_{j} B_{j} \mid X_{j} Y_{j} T_{j}}\left(\cdot, \cdot \mid \cdot, \cdot, t_{j}\right)$ here behaves as $P_{A B \mid X Y}$ does in Sec. III A.

We would like to point out that there is no need to make $\left\{M_{x_{j}}^{a_{j}}\right\}$ (or $\left\{N_{y_{j}}^{b_{j}}\right\}$ ) dependent on previous inputs and outputs, i.e., on $t_{j}$ using the above notation, because we may assume that the measurement $\left\{M_{x_{j}}^{a_{j}}\right\}$ encodes $x_{j}$ and $a_{j}$ into the postmeasurement state of $\mathcal{A}$ and that the subsequent unitary $U_{j+1}$ copies this (classical) information into the state of $\mathcal{B}$. The subsequent measurements can then be control measurements, which perform a measurement depending on the history. Similarly, we may assume the $\left\{M_{x_{j}}^{a_{j}}\right\}$ to be identical for different $j$ (and the same for $\left\{N_{y_{j}}^{b_{j}}\right\}$ ) since the quantum system $\mathcal{A}$ may maintain a counter that is increased by every unitary $U_{j}$, and $\left\{M_{x_{j}}^{a_{j}}\right\}$ can then be chosen as a control measurement that is controlled by the counter. ${ }^{7}$ Given the conditional probability distribution $P_{\boldsymbol{A} B \mid X Y}$ as specified above, which describes the input-output behavior of the $n$ sequential interactions with the device $\mathfrak{D}$, once a distribution $P_{X Y}$ is decided upon, which specifies how the inputs $x_{j}$ and $y_{j}$ are chosen in each round, the joint probability distribution $P_{A B X Y}$ is determined as $P_{A B X Y}=P_{X Y} P_{A B \mid X Y}$.

$b$. Estimating the Bell value. Once the device $\mathfrak{D}$ is given, i.e., the state $\rho_{\mathcal{A B}}$, the measurements $\left(\left\{M_{x_{1}}^{a_{1}}\right\},\left\{N_{y_{1}}^{b_{1}}\right\}\right)$, $\ldots,\left(\left\{M_{x_{n}}^{a_{n}}\right\},\left\{N_{y_{n}}^{b_{n}}\right\}\right)$, and the unitaries $U_{2}, \ldots, U_{n}$ are fixed, $P_{A_{1} B_{1} \mid X_{1} Y_{1}}$ and thus the Bell value of the first round of interaction, $I_{1}=I\left(P_{A_{1} B_{1} \mid X_{1} Y_{1}}\right)$, are determined. For the other rounds, this is slightly more subtle. The reason is that the state $\rho_{\mathcal{A B} \mid T_{2}=t_{2}}$ before the second round, and thus the probability distribution $P_{A_{2} B_{2} \mid X_{2} Y_{2}, T_{2}=t_{2}}$, depends on what happened in the first round, i.e., depends on $t_{2}=\left(a_{1}, b_{1}, x_{1}, y_{1}\right)$. Thus, the Bell value of the second round, $I_{2}=I\left(P_{A_{2} B_{2} \mid X_{2} Y_{2}, T_{2}=t_{2}}\right)$, is a function of $t_{2}$. Similarly, the Bell value of the $j$ th round, $I_{j}=I\left(P_{A_{j} B_{j} \mid X_{j} Y_{j}, T_{j}=t_{j}}\right)$, is a function of $t_{j}$. We let

$$
\bar{I}=\frac{1}{n} \sum_{j=1}^{n} I_{j}
$$

be the average Bell value, averaged over $n$ rounds, and we write $\bar{I}=\bar{I}(\boldsymbol{a}, \boldsymbol{b}, \boldsymbol{x}, \boldsymbol{y})$ to make its dependency on the $\boldsymbol{a}, \boldsymbol{b}$, etc., explicit. $^{8}$

Pironio et al. show in [6] that the average Bell value $\bar{I}$ can be estimated by analyzing the data collected over $n$ rounds.

\footnotetext{
${ }^{7}$ These observations on the independence of the measurements on the history and the round are not crucial for our proofs; they merely simplify the notation.

${ }^{8}$ Actually, it only depends on $\left(a^{n-1}, b^{n-1}, x^{n-1}, y^{n-1}\right)$.
} 
Specifically, defining

$$
\begin{aligned}
\hat{I} & =\hat{I}(\boldsymbol{a}, \boldsymbol{b}, \boldsymbol{x}, \boldsymbol{y}) \\
& =\frac{1}{n} \sum_{j=1}^{n} \sum_{a b x y} c_{a b x y} \frac{\chi\left(a_{j}=a, b_{j}=b, x_{j}=x, y_{j}=y\right)}{P_{X Y}(x, y)},
\end{aligned}
$$

where $\chi(e)$ is the indicator of the event $e$ [that is, $\chi(e)=1$ if the event $e$ occurs and 0 otherwise], the following proposition holds.

Proposition 1 [6]. For $\bar{I}$ and $\hat{I}$ as above, for arbitrary but identically and independently distributed (iid) $(\boldsymbol{X}, \boldsymbol{Y})$, meaning that $P_{X Y}=\prod_{j} P_{X_{j} Y_{j}}$ with $P_{X_{j} Y_{j}}=P_{X Y}$ for all $j$, and for any $\varepsilon>0$,

$$
\begin{aligned}
& P[\bar{I}(\boldsymbol{A}, \boldsymbol{B}, \boldsymbol{X}, \boldsymbol{Y}) \leqslant \hat{I}(\boldsymbol{A}, \boldsymbol{B}, \boldsymbol{X}, \boldsymbol{Y})-\varepsilon] \\
& \quad \leqslant \exp \left(-\frac{\varepsilon^{2} n}{2\left(\frac{c_{\max }}{p_{\min }}+I_{\max }\right)^{2}}\right)
\end{aligned}
$$

where $I_{\max }$ is the maximal value of $I$ achievable by means of a quantum system, $p_{\min }=\min _{x, y} P_{X Y}(x, y)$, and $c_{\max }=$ $\max \left\{c_{a b x y}\right\}$.

Thus, except with small probability, the estimated value $\hat{I}$ for the average Bell value is not much smaller than the real average Bell value $\bar{I}$. For a fixed choice of Bell coefficients $\mathcal{C}=$ $\left\{c_{a b x y}\right\}$, which uniquely determines $I_{\max }$, we write $c\left(p_{\min }\right)=$ $\frac{1}{2 \ln 2}\left(\frac{c_{\max }}{p_{\min }}+I_{\max }\right)^{-2}$, so that the probability in Proposition 1 can be written as $2^{-c\left(p_{\min }\right) \varepsilon^{2} n}$.

We stress that for Proposition 1 to hold, it is crucial that $\boldsymbol{X}$ and $\boldsymbol{Y}$ are chosen independently of the internal state of $\mathfrak{D}$; this is implicit in the statement of Proposition 1 by having modeled the internal state $\rho_{\mathcal{A B}}$ of $\mathfrak{D}$ to be fixed and independent of $\boldsymbol{X}$ and $\boldsymbol{Y}: \rho_{\boldsymbol{X} \boldsymbol{Y} \mathcal{A B}}=\rho_{\boldsymbol{X} \boldsymbol{Y}} \otimes \rho_{\mathcal{A B}}$. Obviously, if $\mathfrak{D}$ knows $\boldsymbol{X}$ and $\boldsymbol{Y}$ in advance, then it can easily be programmed to have a large Bell value while, for instance, being classical.

c. Bounding the min-entropy. It remains to argue that if $\bar{I}$ is nontrivial, i.e., sufficiently greater than $I_{0}$, which can be learned by observing $\hat{I}$ (except with small probability), then the pair $(\boldsymbol{A}, \boldsymbol{B})$ contains a linear (in $n)$ amount of min-entropy. To this end, Pironio et al. show [see Eq. (A5) in [6]] that

$$
P_{A B \mid X Y}(\boldsymbol{a}, \boldsymbol{b} \mid \boldsymbol{x}, \boldsymbol{y}) \leqslant 2^{-h(\bar{I}(\boldsymbol{a}, \boldsymbol{b}, \boldsymbol{x}, \boldsymbol{y}) n)}
$$

for all $\boldsymbol{a}, \boldsymbol{b}, \boldsymbol{x}, \boldsymbol{y}$. In the derivation, they use the fact that $h$ is convex. From (6), they conclude [see Eq. (A9) in [6]] that $H_{\min }(\boldsymbol{A} \boldsymbol{B} \mid \boldsymbol{X}=\boldsymbol{x}, \boldsymbol{Y}=\boldsymbol{y}) \geqslant n h(\bar{I})$ and thus $\geqslant n h(\hat{I}-\varepsilon)$ except with small probability. However, this conclusion does not seem correct. What follows from (6) is that

$$
H_{\min }(\boldsymbol{A} \boldsymbol{B} \mid \boldsymbol{X}=\boldsymbol{x}, \boldsymbol{Y}=\boldsymbol{y}) \geqslant n h\left(\bar{I}\left(a_{0}^{n}, b_{0}^{n}, \boldsymbol{x}, \boldsymbol{y}\right)\right)
$$

for the values $a_{0}^{n}$ and $b_{0}^{n}$ that minimize the right-hand side of (7), but then the right-hand side of (7) is likely to be smaller than $n h(\bar{I}(\boldsymbol{a}, \boldsymbol{b}, \boldsymbol{x}, \boldsymbol{y}))$ or $n h(\hat{I}(\boldsymbol{a}, \boldsymbol{b}, \boldsymbol{x}, \boldsymbol{y}))$ for the values $\boldsymbol{a}$ and $\boldsymbol{b}$ actually observed. ${ }^{9}$

For the remainder of this section, we propose and discuss a possible way to get a meaningful and useful statement on the min-entropy of $(\boldsymbol{A}, \boldsymbol{B})$ in terms of $h(\bar{I})$ and thus of $h(\hat{I})$ except

\footnotetext{
${ }^{9}$ We note that the authors of [6], in a work independent of ours [8], fix this issue in a similar manner as we do here.
}

with small probability. We partition the interval $\left[I_{0}, I_{\max }\right] \subset \mathbb{R}$, ranging from the trivial, meaning classical, Bell value $I_{0}$ to the maximal value $I_{\max }$, into $m$ disjoint blocks: $\left[I_{0}, I_{\max }\right]=\Omega_{0} \cup$ $\cdots \cup \Omega_{m-1}$, where $\Omega_{\ell}$ is of the form $\Omega_{\ell}=\left[J_{\ell}, J_{\ell+1}\right)$, with the exception that $\Omega_{m-1}=\left[J_{m-1}, I_{\max }\right]$ for some boundary points $I_{0}=J_{0} \leqslant J_{1} \leqslant \cdots \leqslant J_{m-1} \leqslant I_{\max }$. The value of $m \in \mathbb{N}$ and the (possibly different) sizes of $\Omega_{\ell}$ are arbitrary but fixed.

For any parameter $\varepsilon>0$, given the random variables $\boldsymbol{A}, \boldsymbol{B}, \boldsymbol{X}, \boldsymbol{Y}$, describing the $n$ interactions with the device $\mathfrak{D}$, we can now define the random variable $L_{\varepsilon}$ to be the unique random variable that satisfies $\hat{I}(\boldsymbol{A}, \boldsymbol{B}, \boldsymbol{X}, \boldsymbol{Y})-\varepsilon \in \Omega_{L_{\varepsilon}}$ (with natural adjustments outside of the range $\left.\left[I_{0}, I_{\max }\right]\right) .^{10}$

Theorem 1. Let $(\boldsymbol{X}, \boldsymbol{Y})$ be iid. Then, for any $\varepsilon, \delta>0$, there exists a "good" event $\mathcal{G}$ with

$$
P[\mathcal{G}] \geqslant 1-m 2^{-\delta n}-3\left(2^{-c\left(p_{\min }\right) \varepsilon^{2} n}\right),
$$

such that

$$
\operatorname{Guess}\left(\boldsymbol{A} \boldsymbol{B} \mid \boldsymbol{X}=\boldsymbol{x}, \boldsymbol{Y}=\boldsymbol{y}, L_{\varepsilon}=\ell, \mathcal{G}\right) \leqslant 2^{-n h\left(J_{\ell}\right)+\delta n+1}
$$

and thus

$$
H_{\min }\left(\boldsymbol{A} \boldsymbol{B} \mid \boldsymbol{X}=\boldsymbol{x}, \boldsymbol{Y}=\boldsymbol{y}, L_{\varepsilon}=\ell, \mathcal{G}\right) \geqslant n h\left(J_{\ell}\right)-\delta n-1
$$

for all $\boldsymbol{x} \in \mathbb{X}^{n}, \quad \boldsymbol{y} \in \mathbb{Y}^{n}$, and $\ell \in\{0, \ldots, m-1\}$ with $P_{\boldsymbol{X Y} L_{\varepsilon} \mid \mathcal{G}}(\boldsymbol{x}, \boldsymbol{y}, \ell)>0$.

We would like to point out that for the bound on $P[\mathcal{G}]$ to hold, it is crucial that $\rho_{\mathcal{A B}}$ is independent of $(\boldsymbol{X}, \boldsymbol{Y})$ [and $\left(X_{i}, Y_{i}\right)$ are iid]: clearly, the device can fool you if it knows the inputs it will get in advance. However, for event $\mathcal{G}$ as defined in the proof below, the bound on the guessing probability holds irrespective of the distribution of $\boldsymbol{X}$ and $\boldsymbol{Y}$. Indeed, the value of $\operatorname{Guess}\left(\boldsymbol{A} \boldsymbol{B} \mid \boldsymbol{X}=\boldsymbol{x}, \boldsymbol{Y}=\boldsymbol{y}, L_{\varepsilon}=\ell, \mathcal{G}\right)$ is determined by the conditional probability distribution $P_{\boldsymbol{A} \boldsymbol{B} \mid \boldsymbol{X} Y}(\cdot, \cdot \mid \boldsymbol{x}, \boldsymbol{y})$ alone (which is determined by $\rho_{\mathcal{A B}}$, the family of measurements and the unitaries); this holds because $L_{\varepsilon}$ as well as $\mathcal{G}$ (we will see this below) are uniquely determined by $\boldsymbol{A}, \boldsymbol{B}, \boldsymbol{X}$, and $\boldsymbol{Y}$.

Proof. Let $\mathcal{B}^{\text {guess }}$ be the bad event $\bar{I}(\boldsymbol{A}, \boldsymbol{B}, \boldsymbol{X}, \boldsymbol{Y}) \leqslant$ $\hat{I}(\boldsymbol{A}, \boldsymbol{B}, \boldsymbol{X}, \boldsymbol{Y})-\varepsilon$ where the estimated Bell value $\hat{I}$ is significantly larger than the average Bell value $\bar{I}$, and let $\mathcal{G}^{\text {guess }}$ be its complement (which we understand as a good event); by Proposition 1 , we know that $P\left[\mathcal{B}^{\text {guess }}\right] \leqslant 2^{-c\left(p_{\min }\right) \varepsilon^{2} n}$. We define $\mathcal{B}_{1}$ to be the set of all "bad inputs" $(\boldsymbol{x}, \boldsymbol{y})$ with the property

$$
P\left[\mathcal{B}^{\text {guess }} \mid \boldsymbol{X}=\boldsymbol{x}, \boldsymbol{Y}=\boldsymbol{y}\right] \geqslant \frac{1}{2} ;
$$

it is straightforward to show that $P\left[(\boldsymbol{X}, \boldsymbol{Y}) \in \mathcal{B}_{1}\right] \leqslant$ $2\left(2^{-c\left(p_{\min }\right) \varepsilon^{2} n}\right)$. Finally, we define $\mathcal{B}_{2}$ to be the set of all $(\boldsymbol{x}, \boldsymbol{y}, \ell)$ with the property

$$
P_{L_{\varepsilon} \mid X Y \mathcal{G}^{\text {guess }}}(\ell \mid \boldsymbol{x}, \boldsymbol{y}) \leqslant 2^{-\delta n} .
$$

It follows from the definition of $\mathcal{B}_{2}$ that

$$
P\left[\left(\boldsymbol{X}, \boldsymbol{Y}, L_{\varepsilon}\right) \in \mathcal{B}_{2} \mid \mathcal{G}^{\text {guess }}\right] \leqslant m 2^{-\delta n} .
$$

We slightly abuse notation and identify the set $\mathcal{B}_{1}$ with the bad event $(\boldsymbol{X}, \boldsymbol{Y}) \in \mathcal{B}_{1}$, and we write $\mathcal{G}_{1}$ for its complementary

\footnotetext{
${ }^{10}$ The definition of $L_{\varepsilon}$ simply captures that if $\hat{I}$ is too close to the lower end of an interval, then we take the next lower interval to be on the safe side.
} 
good event and correspondingly for $\mathcal{B}_{2}$ and $\mathcal{G}_{2}$. We now define the good event $\mathcal{G}$ as $\mathcal{G}:=\mathcal{G}^{\text {guess }} \wedge \mathcal{G}_{1} \wedge \mathcal{G}_{2}$. Using the union bound over the bad events, it is not too hard to show that $P[\mathcal{G}] \geqslant 1-m 2^{-\delta n}-3\left(2^{-c\left(p_{\min }\right) \varepsilon^{2} n}\right)$.

It remains to argue the bound on the min-entropy. Let $\boldsymbol{a}, \boldsymbol{b}, \boldsymbol{x}, \boldsymbol{y}$ be such that $\bar{I}(\boldsymbol{a}, \boldsymbol{b}, \boldsymbol{x}, \boldsymbol{y})>\hat{I}(\boldsymbol{a}, \boldsymbol{b}, \boldsymbol{x}, \boldsymbol{y})-\varepsilon$; i.e., they have positive probability conditioned on the good event $\mathcal{G}^{\text {guess }}$. Furthermore, let $\ell$ be the unique value with

$$
\hat{I}(\boldsymbol{a}, \boldsymbol{b}, \boldsymbol{x}, \boldsymbol{y})-\varepsilon \in \Omega_{\ell} .
$$

If $(\boldsymbol{x}, \boldsymbol{y}) \notin \mathcal{B}_{1}$, then $P\left[\mathcal{G}^{\text {guess }} \mid \boldsymbol{X}=\boldsymbol{x}, \boldsymbol{Y}=\boldsymbol{y}\right] \geqslant \frac{1}{2}$, and hence, conditioning on the event $\mathcal{G}^{\text {guess }}$ can increase the probabilities by at most a factor of 2 . For those $(\boldsymbol{x}, \boldsymbol{y}) \notin \mathcal{B}_{1}$, it then follows from (6) that

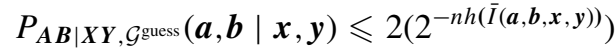

$$
\begin{aligned}
& \leqslant 2\left(2^{-n h(\hat{I}(\boldsymbol{a}, \boldsymbol{b}, \boldsymbol{x}, \boldsymbol{y})-\varepsilon)}\right) \\
& \leqslant 2\left(2^{-n h\left(J_{\ell}\right)}\right) \text {. }
\end{aligned}
$$

If additionally we have $(\boldsymbol{x}, \boldsymbol{y}, \ell) \notin \mathcal{B}_{2}$, then

$$
\begin{aligned}
P_{\boldsymbol{A} \boldsymbol{B} \mid \boldsymbol{X} \boldsymbol{Y} L_{\varepsilon}, \mathcal{G}^{\text {guess }}}(\boldsymbol{a}, \boldsymbol{b} \mid \boldsymbol{x}, \boldsymbol{y}, \ell) & \leqslant \frac{P_{\boldsymbol{A} \boldsymbol{B} \mid \boldsymbol{X} \boldsymbol{Y}, \mathcal{G}^{\text {guess }}}(\boldsymbol{a}, \boldsymbol{b} \mid \boldsymbol{x}, \boldsymbol{y})}{P_{L_{\varepsilon} \mid \boldsymbol{X} \boldsymbol{Y} \mathcal{G}^{\text {guess }}}(\ell \mid \boldsymbol{x}, \boldsymbol{y})} \\
& \leqslant 2\left(2^{-n h\left(J_{\ell}\right)}\right)\left(2^{\delta n}\right) .
\end{aligned}
$$

Note that additionally conditioning on $\mathcal{G}_{1}$ and $\mathcal{G}_{2}$ does not change the above conditional probability distribution if $(\boldsymbol{x}, \boldsymbol{y}) \notin \mathcal{B}_{1}$ and $(\boldsymbol{x}, \boldsymbol{y}, \ell) \notin \mathcal{B}_{2}$. Thus, the same bound also applies to $P_{\boldsymbol{A} \boldsymbol{B} \mid \boldsymbol{X} Y L_{\varepsilon}, \mathcal{G}}(\boldsymbol{a}, \boldsymbol{b} \mid \boldsymbol{x}, \boldsymbol{y}, \ell)$ for all $\boldsymbol{a}, \boldsymbol{b}, \boldsymbol{x}, \boldsymbol{y}$, and $\ell$ with $P_{\boldsymbol{A} \boldsymbol{B} \boldsymbol{X} \boldsymbol{Y} L_{\varepsilon} \mid \mathcal{G}}(\boldsymbol{a}, \boldsymbol{b}, \boldsymbol{x}, \boldsymbol{y}, \ell)>0$. By definition of the guessing probability and the min-entropy, this proves the claim.

d. A specific example. Consider $\mathrm{CHSH}$, so that the Bell value of a given device is expected to be in the range from $I_{0}=2$ to $I_{\max }=2 \sqrt{2} \approx 2.828$. Let us divide this range into $J_{0}=I_{0}<$ $J_{1}=2.2<J_{2}=2.4<J_{3}=2.6<I_{\max }$, and let us take a $q$ biased input distribution $P_{X Y}=\prod_{j} P_{X_{j} Y_{j}}$ with $P_{X_{j} Y_{j}}(0,0)=$ $1-3 q$ and $P_{X_{j} Y_{j}}(x, y)=q$ for all $(x, y) \in\{0,1\}^{2} \backslash\{(0,0)\}$, where $0<q \leqslant 1 / 4$ is some parameter. Finally, let us fix some small parameters $\varepsilon, \delta>0$; for concreteness, say that $\varepsilon=0.05$ and $\delta=0.01$.

Consider now $n$ sequential interactions with an untrusted device $\mathfrak{D}$, where in each round $x_{j}$ and $y_{j}$ are chosen (according to $P_{X_{j} Y_{j}}$ ) and input into $\mathfrak{D}$, and $a_{j}$ and $b_{j}$ are obtained as output from $\mathfrak{D}$. Let us say that from the collected data, we get $\hat{I}(\boldsymbol{a}, \boldsymbol{b}, \boldsymbol{x}, \boldsymbol{y})=2.7 \in \Omega_{3}$ as the estimation for the average Bell value. By Theorem 1, we have that, given $\boldsymbol{x}$ and $\boldsymbol{y}$ and $L_{\varepsilon}=3$, the min-entropy of $\boldsymbol{a}$ and $\boldsymbol{b}$ is at least $n[h(2.6)-\delta]-1 \approx n(0.36-\delta)>n / 3$ bits, except with probability $4\left(2^{-\delta n}\right)+3\left(2^{-c(q) \varepsilon^{2} n}\right) .{ }^{11}$ Thus, when applying a suitable randomness extractor to $\boldsymbol{a}, \boldsymbol{b}$, we can extract, say, $n / 4$ bits that are exponentially close to uniformly distributed (given $\boldsymbol{x}$ and $\boldsymbol{y}$ and $L_{\varepsilon}=3$ ).

In order to sample the inputs according to the biased input distribution $P_{X Y}$, as suggested in [6], it is known to be sufficient (on average) to have access to $n O\left(q \log _{2}(1 / q)\right)$

\footnotetext{
${ }^{11}$ This probability is an average value over $\boldsymbol{A} \boldsymbol{B}, \boldsymbol{X} \boldsymbol{Y}$.
}

random bits [14]. Since $q \log _{2}(1 / q)$ converges to 0 for $q \rightarrow 0$, if $q$ is chosen to be a small enough constant, then, say, $n / 4$ random bits are sufficient. Thus, by starting off with $n / 4$ random bits, we obtained another $n / 4$ almost-random bits and thus hold now $n / 2$ random bits. ${ }^{12}$ Thus, we have expanded the randomness by a factor 2 . Choosing $q=O\left(n^{-1 / 3}\right)$, one obtains an expansion factor $O\left(n^{2 / 3} / \log _{2} n\right)$ while still being negligibly close to perfect randomness [since $c\left(n^{-1 / 3}\right)=\Omega\left(n^{-2 / 3}\right)$ ].

Having generated fresh randomness from an untrusted device $\mathfrak{D}$, one is now tempted to use the newly obtained randomness to generate even more fresh randomness from the device $\mathfrak{D}$ and so on. This does not work. The reason is that the generated randomness is not random to the device $\mathfrak{D}$, or, more formally, not independent of the internal state of $\mathfrak{D}$; indeed, $\mathfrak{D}$ has already observed $\boldsymbol{x}$ and $\boldsymbol{y}$, and it has itself produced $\boldsymbol{a}$ and $\boldsymbol{b}$. We argue below, however, that we can use the fresh randomness to generate even more randomness from another device, as long as the devices are not entangled with each other or with the adversary.

e. Classical side information. The case where the adversarial producer of the devices Eve holds classical side information about the device $\mathfrak{D}$ can be reduced to the case without side information by conditioning on particular values of the side information.

f. Quantum side information? Ideally, one would like to obtain similar results in the case where Eve holds quantum side information about the device $\mathfrak{D}$, i.e., where Eve maintains a quantum state $\mathcal{E}$ that is entangled with state $\mathcal{A B}$ contained in $\mathfrak{D}$. It is not too hard to see that if the function $2^{-h(\cdot)}$ is concave (which is, e.g., satisfied for $\mathrm{CHSH}$ ), then the minentropy bound $H_{\min }(A B \mid X=x, Y=y) \geqslant h(I)$ for a single interaction, which holds by definition of the function $h$, extends to the case of quantum side information in that also $H_{\text {min }}(A B \mid X=x, Y=y, \mathcal{E}) \geqslant h(I)$ holds, where $I$ is the Bell value of the distribution obtained by tracing out $\mathcal{E}$. This seems to suggest that quantum side information is useless for Eve.

Unfortunately, the techniques we use for analyzing sequential repetitions do not appear applicable in the case of quantum side information. The main technical problem we run into is to show that a lower bound on the smooth min-entropy of a random variable conditioned on a quantum state can be obtained from a lower bound on the smooth min-entropy of the random variable conditioned on the measurement outcome for every possible measurement (as is the case for the nonsmooth version [10]). ${ }^{13}$ It is not clear if such a statement is actually true.

Thus, whether quantum side information is useless for Eve also in the case of sequential repetition, where the average Bell value is estimated from the observed data, remains an open problem. We refer to [7] for a different proof technique which does work against quantum side information.

\footnotetext{
${ }^{12} \mathrm{We}$ are ignoring here the randomness needed for the extractor.

${ }^{13}$ Essentially, the notion of smooth min-entropy takes into account that we may condition on a good event that has a probability close to 1 .
} 
Let $\varepsilon, \delta, e>0$ be the security parameters. Let $t>0$ and assume long enough initial randomness $K_{\text {init }}=\left(K_{0}, K_{\mathrm{Bank}}\right)$, split into parts of size $\left|K_{0}\right|=\eta$ and $\left|K_{\mathrm{Bank}}\right|=O\left(t \eta^{2} \log _{2} \eta\right)$. Denote the second part by $K_{\mathrm{Bank}}=S_{1} \cdots S_{t}$ where $\left|S_{1}\right|=\cdots=\left|S_{t}\right|=O\left(\eta^{2} \log _{2} \eta\right)$.

1. For $i=1$ to $t$ do:

(a) Set $R_{i}=$ Sample $\left(K_{i-1}\right)$.

(b) Input $R_{i}$ to $\mathfrak{D}_{(i \bmod 2)}$ and denote the output by $W_{i}$.

(c) Estimate the Bell value $\hat{I}_{i}$ of the $i$ th interaction with the devices [Eq. (5)], and determine the unique value $\ell_{i}$ that satisfies $\hat{I}_{i}-\varepsilon \in \Omega_{\ell_{i}}$

(d) Calculate the guaranteed min-entropy in $W_{i}, \mathrm{H}_{\min }^{i}:=\left\|W_{i}\right\|\left(\mathrm{h}\left(J_{\ell_{i}}\right)-\delta\right)-1$, and let $\xi_{i}=\left\lfloor\mathrm{H}_{\min }^{i}-4 \log _{2} \varepsilon_{\text {ext }}^{-1}-c_{\text {ext }}\right\rfloor$, with a constant $c_{\text {ext }}$ specified in Lemma 1.

(e) If $\xi_{i} \leq \eta$, set $K_{i}=K_{i+1}=\ldots=K_{t}=\perp$ (the empty string) and go to step 2 . Otherwise set $K_{i}=\operatorname{Ext}\left(W_{i}, S_{i}\right)\left(\right.$ with $\varepsilon_{\text {ext }}=2^{-e \eta}$ and $\left.\xi_{i}=\left|K_{i}\right|\right)$.

2. Output $K_{t}$.

FIG. 1. Randomness expansion scheme with two independent devices.

\section{COMPOSABILITY}

Consider two (or more) untrusted devices $\mathfrak{D}$ and $\mathfrak{D}^{\prime}$, prepared by the adversary Eve. We assume that $\mathfrak{D}$ and $\mathfrak{D}^{\prime}$ cannot communicate and are not entangled with each other. The case when Eve holds classical side information about the devices can be treated as described in the previous section. We can then apply Theorem 1 to argue that the output $\boldsymbol{A} \boldsymbol{B}$ produced by $\mathfrak{D}$ has high min-entropy (except with small probability) given the internal state of $\mathfrak{D}^{\prime}$ (because $\mathfrak{D}^{\prime}$ is independent of $\mathfrak{D}$ ), assuming that a large enough average Bell value is observed. It thus follows that by applying an extractor (with suitable parameters and a freshly chosen seed) to $\boldsymbol{A B}$, we obtain a bit string $K$ that is close to random and independent of the internal state of $\mathfrak{D}^{\prime}$. This in particular implies that if we use the randomness $K$ to sample the input $\boldsymbol{X}^{\prime} \boldsymbol{Y}^{\prime}$ to $\mathfrak{D}^{\prime}$ (according to a prescribed distribution), then $\boldsymbol{X}^{\prime} \boldsymbol{Y}^{\prime}$ is close to independent of the internal state of $\mathfrak{D}^{\prime}$. As the dependency between the internal (quantum) state of $\mathfrak{D}$ and the inputs and outputs of $\mathfrak{D}^{\prime}$ is purely classical, we can condition on this classical information and apply Theorem 1 to argue that the output $\boldsymbol{A}^{\prime} \boldsymbol{B}^{\prime}$ produced by $\mathfrak{D}^{\prime}$ has high min-entropy given the current internal state of $\mathfrak{D}$. Therefore, we are in the same situation as above and so can use the randomness extracted from $\boldsymbol{A}^{\prime} \boldsymbol{B}^{\prime}$ to sample again inputs for $\mathfrak{D}$, and we can keep on going like this as long as a large enough Bell value is observed. We stress that the above line of reasoning only works because we assumed the devices $\mathfrak{D}, \mathfrak{D}^{\prime}$ to be unentangled to start with.

\section{A. Randomness expansion with independent devices}

We increase the level of abstraction and from now on consider an untrusted device $\mathfrak{D}$ as an abstract object that takes some input $R=(\boldsymbol{X}, \boldsymbol{Y})=\left(X^{n}, Y^{n}\right)$ and produces some output $W=(\boldsymbol{A}, \boldsymbol{B})=\left(A^{n}, B^{n}\right)$ and maintains some internal quantum state $\mathcal{E}_{\mathfrak{D}}$. It is guaranteed that, if $R$ is properly distributed and $W$ satisfies some statistical property, then $W$ has lower-bounded min-entropy given $R=r$ (except with small probability), even given classical side information. Let $|R|$ denote the length of the bit string $R$, that is, $|R|=n \log _{2}|\mathbb{X}||\mathbb{Y}| \quad$ (similarly, $|W|=n \log _{2}|\mathbb{A}||\mathbb{B}|$ ). Let $\|R\|=n$ be the number of pairs $(x, y)$ in $R$, that is, the number of sequential interactions with the device caused by the input $R$ (respectively, $\|W\|=n$ ).

Instead of using $v$ devices, our scheme alternates between two devices $\mathfrak{D}_{0}, \mathfrak{D}_{1}$. Formally (Fig. 1), we split the initial randomness $K_{\text {init }}$ into two parts $K_{\text {init }}=\left(K_{0}, K_{\text {Bank }}\right)$ such that $\left|K_{0}\right|=\eta$ and $\left|K_{\text {Bank }}\right|=O\left(t \eta^{2} \log _{2} \eta\right)$ for some constant $t>$ 0 . The first part $K_{0}$ is used to generate an input to the devices, and the other part $K_{\text {Bank }}$ is used as a "bank" of $t$ seeds for the extractor. For $i=1, \ldots, t$, let $R_{i}$ denote the input given to the device on the $i$ th activation. ${ }^{14}$ For the first iteration, the input is the biased string $R_{1}=\operatorname{Sample}\left(K_{0}\right)$. Let $W_{i}$ denote the output of the $i$ th activation. In order to generate the input for the next iteration, $R_{i+1}$, we use an extractor on the bit string $W_{i}$ with a fresh random seed $S_{i}$ from the $K_{\text {Bank }}$. Denote the extracted string by $K_{i}=\operatorname{Ext}\left(W_{i}, S_{i}\right)$. Finally, $K_{i}$ is used to generate a biased string $R_{i+1}=\operatorname{Sample}\left(K_{i}\right)$, which is the input for the next iteration. Note that each iteration achieves at most quadratic expansion; hence the strings $R_{i}, W_{i}, K_{i}$ are of length $\operatorname{poly}(\eta)$.

In order to extract randomness out of $W_{i}$, we use the extractor of De et al. [15] with $\varepsilon_{\text {ext }}=2^{-e \eta}$, where the constant $e>0$ is taken arbitrarily small.

Lemma 1 (Corollary 5.2 in [15]). There exists an extractor Ext $:\{0,1\}^{n} \times\{0,1\}^{d} \rightarrow\{0,1\}^{\xi}$ which is

$$
\left(\xi+4 \log _{2} \varepsilon_{\text {ext }}^{-1}+c_{\text {ext }}, \varepsilon_{\text {ext }}\right)
$$

strong against quantum adversaries, with $c_{\text {ext }}=O(1)$ and a seed of size $d=O\left(\log _{2}^{2}\left(n / \varepsilon_{\text {ext }}\right) \log _{2} \xi\right)$.

\footnotetext{
${ }^{14}$ We alternate between the devices; thus the $i$ th activation is done with $\mathfrak{D}_{(i \bmod 2)}$, which is also referred to as the $i$ th device.
} 
The seed for the extractor is of length

$$
\begin{aligned}
& O\left(\log _{2}^{2}\left(\left|W_{i}\right| / \varepsilon_{\text {ext }}\right) \log _{2} \xi_{i}\right) \\
& \quad=O\left(\left[\log _{2}^{2} \operatorname{poly}(\eta)+e^{2} \eta^{2}\right] \log _{2} \operatorname{poly}(\eta)\right) \\
& \quad=O\left(e^{2} \eta^{2} \log _{2} \eta\right) .
\end{aligned}
$$

\section{CONCLUSION AND OPEN PROBLEMS}

An interesting extension to our result is to generalize Theorem 1 to the setting of quantum side information. This would allow a composition theorem for the more general case in which the devices can be entangled with each other and with
Eve. Although we can allow quantum side information for $a$ single interaction with the device, we are currently unable to give a rigorous proof of security against quantum side information for multiple interactions and leave it as the main open question.

\section{ACKNOWLEDGMENTS}

R.G. is grateful to CWI, Amsterdam, for hosting him while part of this work was done. C.S. is supported by a NWO VENI grant. This manuscript was made open-access thanks to a grant by NWO.
[1] A. Einstein, B. Podolsky, and N. Rosen, Phys. Rev. 47, 777 (1935).

[2] J. Bell, Physics 1, 195 (1964).

[3] J. F. Clauser, M. A. Horne, A. Shimony, and R. A. Holt, Phys. Rev. Lett. 23, 880 (1969).

[4] R. Colbeck, Ph.D. thesis, University of Cambridge, 2009.

[5] R. Colbeck and A. Kent, J. Phys. A 44, 095305 (2011).

[6] S. Pironio, A. Acín, S. Massar, A. B. de la Giroday, D. N. Matsukevich, P. Maunz, S. Olmschenk, D. Hayes, L. Luo, T. A. Manning, and C. Monroe, Nature (London) 464, 1021 (2010).

[7] U. Vazirani and T. Vidick, Philos. Trans. R. Soc. A 370, 3432 (2012).

[8] S. Pironio and S. Massar, Phys. Rev. A 87, 012336 (2013).
[9] J. Barrett, R. Colbeck, and A. Kent, Phys. Rev. Lett. 110, 010503 (2013).

[10] R. König, R. Renner, and C. Schaffner, IEEE Trans. Inf. Theory 55, 4337 (2009).

[11] R. Renner, Ph.D. thesis, ETH Zürich, 2005.

[12] M. Navascués, S. Pironio, and A. Acín, Phys. Rev. Lett. 98, 010401 (2007)

[13] M. Navascués, S. Pironio, and A. Acín, New J. Phys. 10, 073013 (2008).

[14] D. Knuth and A. Yao, in Algorithms and Complexity: New Directions and Recent Results (Academic, New York, 1976), pp. 357-428.

[15] A. De, C. Portmann, T. Vidick, and R. Renner, SIAM J. Comput. 41, 915 (2012). 